\title{
BMJ Open Access and barriers to healthcare in the Kingdom of Saudi Arabia, 2013: findings from a national multistage survey
}

\author{
Charbel El Bcheraoui, ${ }^{1}$ Marwa Tuffaha, ${ }^{1}$ Farah Daoud, ${ }^{1}$ Hannah Kravitz, ${ }^{1}$ \\ Mohammad A AlMazroa, ${ }^{2}$ Mohammad Al Saeedi, ${ }^{2}$ Ziad A Memish, ${ }^{2}$ \\ Mohammed Basulaiman, ${ }^{2}$ Abdullah A Al Rabeeah, ${ }^{2}$ Ali H Mokdad ${ }^{1}$
}

To cite: El Bcheraoui $C$, Tuffaha M, Daoud F, et al. Access and barriers to healthcare in the Kingdom of Saudi Arabia, 2013: findings from a national multistage survey. BMJ Open 2015;5: e007801. doi:10.1136/ bmjopen-2015-007801

- Prepublication history for this paper is available online To view these files please visit the journal online (http://dx.doi.org/10.1136/ bmjopen-2015-007801)

Received 28 January 2015 Revised 1 April 2015 Accepted 8 May 2015
CrossMark

\footnotetext{
${ }^{1}$ Institute for Health Metrics and Evaluation, University of Washington, Seattle, Washington, USA ${ }^{2}$ Ministry of Health of the Kingdom of Saudi Arabia, Riyadh, Saudi Arabia
}

Correspondence to Dr Ali H Mokdad; mokdaa@uw.edu

\section{ABSTRACT}

Objectives: We analysed data from a large household survey to identify barriers to healthcare in the Kingdom of Saudi Arabia.

Methods: The Saudi Health Interview Survey (SHIS) is a national multistage survey of individuals aged 15 years or older. The survey combined a household questionnaire and a laboratory blood analysis. We used a backward elimination multivariate logistic regression model to measure association between (1) diagnosis, (2) treatment, and (3) control of hypertension or diabetes and sociodemographic factors, history of diagnosis with chronic conditions, and type of, and distance travelled to, the clinic last visited.

Results: Between April and June 2013, a total of 10735 participants completed SHIS and were invited to the local health clinics. Among hypertensive individuals, women, older individuals, and those previously diagnosed with diabetes and hypercholesterolaemia were more likely to have been diagnosed with hypertension than their counterparts. Among participants diagnosed with hypertension, the likelihood of being treated increased with age and education. The likelihood of having uncontrolled blood pressure despite treatment increased with education and a history of diagnosis with hypercholesterolaemia. Type of clinic visited and distance travelled to last clinic visit were not associated with diagnosis or treatment of hypertension or control of blood pressure. Similar factors were associated with the likelihood of diagnosis and treatment among individuals with diabetes. Having uncontrolled glycated haemoglobin levels, despite treatment, was less common among those who visited governmental clinics other than those of the Ministry of Health, compared with those who visited Ministry clinics.

Conclusions: Our findings highlight the importance of individual characteristics in healthcare-seeking practices rather than system-based potential barriers. Saudis seem to mostly seek healthcare when sick. Hence, the Saudi Ministry of Health needs to implement a comprehensive plan including health education and investigations, to understand the barriers and bottlenecks to healthcare-seeking behaviour.

\section{Strengths and limitations of this study}

- First nationally representative study on access and barriers to healthcare in the Kingdom of Saudi Arabia.

- Highlights the importance of individual, over environmental and systematic, characteristics in healthcare-seeking behaviour among Saudis.

- Calls for in-depth investigations on beliefs and attitudes affecting healthcare-seeking behaviour among Saudis.

- Cross-sectional design limits assessment of causality.

- Lowered response rate for laboratory testing.

\section{INTRODUCTION}

In the Kingdom of Saudi Arabia (KSA), healthcare is offered for free to Saudi citizens ${ }^{1}$ through more than 2000 primary healthcare centres and 420 hospitals. ${ }^{2}$ We have previously reported that Saudis do not make use of periodic health examinations despite their cost-free availability. ${ }^{3}$ In a more specific example, we have also reported that among women eligible for breast cancer screening, only a low percentage are actually screened. ${ }^{4}$

The Saudi Ministry of Health is investing considerable effort and money to prevent disease and promote health, with a special focus on non-communicable diseases. However, despite these efforts, funds and the free healthcare system in KSA, more than 1.9 million (15.2\%) and 1.7 million (13.4\%) Saudis aged 15 years or older are hypertensive and diabetic, respectively. ${ }^{5}$ Furthermore, $57.8 \%$ and $43.6 \%$ of those affected have not been diagnosed with hypertension and diabetes, respectively, while among those diagnosed, $31.1 \%$ and $9 \%$ are not treated. Among those treated, $55.0 \%$ and $29.1 \%$ do not have their condition under control.

Distance to healthcare settings and types of healthcare settings have been reported to impact on patients' healthcare-seeking behaviour. $^{7-10}$ 
To identify barriers to healthcare in KSA, we analysed data from a large household survey on the relation of distance travelled to healthcare clinics and type of clinics last visited, and compared these data with diagnosis, treatment, and control of hypertension and diabetes.

\section{METHODS}

The Saudi Health Interview Survey (SHIS) is a national multistage survey of individuals aged 15 years or older. Households of Saudi citizens were randomly selected from a national sampling frame maintained and updated by the Census Bureau. The Ministry of Health divides KSA into 13 health regions, each with its own health department. We divided each region into subregions and blocks used by the KSA Department of Statistics. All regions were included, and a probability proportional to size was used to randomly select subregions and blocks. Households were randomly selected from each block. We used a computer-assisted personal interviewing methodology for data collection. A roster of all household members was collected, and an adult aged 15 or older was selected to be surveyed through a simple random sampling algorithm programmed in the computers used for data collection. If the randomly selected adult was not present, our surveyors made an appointment to return, and a total of three visits were made before the household was considered as a non-response. Blood pressure of the randomly selected adult was measured at the household by a trained professional.

The survey included questions on sociodemographic characteristics, healthcare utilisation and self-reported chronic conditions. These conditions included diabetes, hypercholesterolaemia and hypertension.

To measure diagnosed hypertension, diabetes and hypercholesterolaemia status, respondents were asked three separate questions: "Have you ever been told by a doctor, nurse, or other health professional that you had (1) diabetes mellitus, otherwise known as diabetes, sugar diabetes, high blood glucose, or high blood sugar; (2) hypercholesterolaemia, otherwise known as high or abnormal blood cholesterol; (3) hypertension, otherwise known as high blood pressure?". Women diagnosed with diabetes or hypertension during pregnancy were not counted as having these conditions.

A total of three blood measurements were taken with the participant resting and at 5 min intervals. We followed National Health and Nutrition Examination Survey (NHANES) guidelines for determining blood pressure level. ${ }^{11}$ Respondents were considered to have hypertension if they met any of the following criteria: (1) measured diastolic or systolic blood pressure exceeding 89 or $139 \mathrm{~mm} \mathrm{Hg}$, respectively, or (2) measured diastolic or systolic blood pressure not exceeding the appropriate threshold, but where the respondent reported taking medications for hypertension. Hence, respondents who were on drugs for hypertension were considered hypertensive even if their measured diastolic or systolic blood pressure did not exceed 89 or $139 \mathrm{~mm} \mathrm{Hg}$, respectively.

Respondents who completed the questionnaire were invited to local primary healthcare clinics to provide a blood sample for laboratory analysis. All blood samples were analysed in a central laboratory at the King Fahd Medical City in Riyadh. COBAS INTEGRA400 plus was used to measure blood levels of glycated haemoglobin (HbA1C). We followed NHANES guidelines for determining diabetes status. ${ }^{11}$ Respondents were considered to be diabetic if they met any of the following criteria: (1) measured HbA1c equalling or exceeding $6.5 \%$ ( $48.5 \mathrm{mmol} / \mathrm{mol}$ ) or (2) measured HbA1c not equalling or exceeding $6.5 \%(48.5 \mathrm{mmol} / \mathrm{mol})$, but where the respondent reported taking medications for diabetes. Hence, the subgroup 'diabetic' includes those with measured HbAlc equal to or above $6.5 \%$ or those taking medication for diabetes.

To assess use of healthcare services, participants were asked, "What was the type of the clinic that you last visited for medical attention for any reason?" and "How far away was the facility you attended from your home?".

\section{Statistical analysis}

We used a backward elimination multivariate logistic regression model to measure association between (1) diagnosis, (2) treatment and (3) control of hypertension or diabetes, and sex, age, marital status, education, history of diagnosis with diabetes, hypercholesterolaemia, hypertension, and type of, and distance travelled to, the clinic last visited. The logistic regression eliminated missing data. Data were weighted to account for the probability of selection, and age and sex poststratification based on census data, for age and sex distribution of the Saudi population.

\section{Weighting methodology}

Two sets of sampling weights were generated and incorporated into the data set for analysis.

First, we created an individual sampling weight for all respondents to account for (1) the probability of selection of an eligible respondent within a household, (2) the probability of selection of the household within a stratum, and (3) the post-stratification differences in age and sex distribution, between the sample and the Saudi population.

For individuals who completed the laboratory-based blood analysis, we computed an additional sampling weight used in analysing data from clinic visits to account for (1) the individual sampling weight described above, (2) the probability of visiting a clinic, (3) sociodemographic, behavioural and health differences between respondents who visited the clinic and those who did not, and (4) the post-stratification differences in age and sex distribution between the respondents who visited the clinic, and the Saudi population. We used SAS 9.3 for the analyses and to account for the complex sampling design. 


\section{RESULTS}

Between April and June 2013, a total of 10735 participants completed the SHIS-a response rate of $89.4 \%$ and were invited to the local health clinics. The remaining 1265 completed part of the household roster or all of it, but when an adult was randomly selected, this adult did not complete the survey.

An estimated total of 1957191 (15.2\%) Saudis aged 15 years or older had hypertension (measured or reported taking blood pressure medication). Of these, 1119027 were undiagnosed. Women (adjusted OR $(\mathrm{AOR})=1.87 ; 95 \%$ CI 1.23 to 2.85), older individuals (AOR=1.05; 95\% CI 1.03 to 1.07 ), and those previously diagnosed with diabetes $(\mathrm{AOR}=2.38 ; 95 \%$ CI 1.60 to $3.53)$ and hypercholesterolaemia (AOR $=2.76 ; 95 \%$ CI 1.72 to 4.44 ), were more likely to have been diagnosed with hypertension than men, younger individuals and those without such a history of diagnosis (table 1).

Among participants diagnosed with hypertension, $78.9 \%$ reported taking medication for their condition. The likelihood of being treated increased with age (AOR=1.08; 95\% CI 1.05 to 1.12) and among those who completed elementary or high school $(\mathrm{AOR}=2.86 ; 95 \%$ CI 1.16 to 7.08$)$ or college (AOR=2.78; 95\% CI 1.14 to 6.79) (table 1).

About $45 \%$ of participants on medication for hypertension had their blood pressure controlled. Hence, about 390338 adults had uncontrolled blood pressure. The likelihood of having uncontrolled blood pressure despite treatment increased among those who completed elementary or high school $(\mathrm{AOR}=2.25 ; 95 \%$ CI 1.12 to 4.50$)$ and college ( $\mathrm{AOR}=4.63 ; 95 \%$ CI 2.14 to 10.02 ) and those previously diagnosed with hypercholesterolaemia (AOR=1.89; 95\% CI 1.12 to 3.18 ).

Type of clinic visited and distance travelled to last clinic visit were not associated with diagnosis or treatment of hypertension or control of blood pressure.

A total of 5590 individuals went to the local clinics and provided blood samples for analyses-a response rate of $52.1 \%$. A total of $1745532(13.4 \%)$ Saudis aged 15 years or older had diabetes. Among those whom our survey identified as diabetic from blood examinations, $43.6 \%$ were undiagnosed. Older individuals $(\mathrm{AOR}=1.05$; $95 \%$ CI 1.03 to 1.08 ) and those previously diagnosed with hypertension (AOR=2.39; 95\% CI 1.09 to 5.25) and hypercholesterolaemia $\quad(\mathrm{AOR}=5.64 ; 95 \%$ CI 2.37 to 13.44) were more likely to be diagnosed than younger individuals and those without such a history of diagnosis (table 1).

Among participants diagnosed with diabetes, 91\% reported taking medication for their condition. The likelihood of being treated increased among those with a college degree (AOR=5.79; 95\% CI 1.57 to 21.32) (table 2).

About $70.9 \%$ of participants on medication for diabetes had their diabetes controlled. Hence, about 397541 adults had uncontrolled diabetes. The likelihood of having uncontrolled levels of HbAlc despite treatment decreased among those who visited governmental clinics other than those of the Ministry of Health (AOR $=0.28 ; 95 \%$ CI 0.10 to 0.79 ), compared with those who visited the Ministry clinics (table 2).

\section{DISCUSSION}

This is the first national study to examine barriers to healthcare utilisation in KSA. Our findings highlight the importance of individual characteristics in healthcareseeking practices, rather than system-based potential barriers. Our results show that neither distance to nor type of healthcare clinic were barriers to diagnosis, treatment, or control of two leading chronic disease in KSA. Our findings are of great importance, as the Ministry continues to invest in building infrastructure throughout the Kingdom. The 2014 Ministry of Health budget calls for building 34 new hospitals. ${ }^{12}$ Therefore, it is crucial to assess the Saudis' reasons for not seeking care, and to address this aspect in order to improve health and reduce burden.

Access to healthcare is an important determinant of health. Several studies have shown that the availability of facilities within accessible distance improves health. Therefore, in some countries, including South Africa, KSA and Portugal, distance to key populations is considered when planning on building new health facilities. ${ }^{13-15}$ Saeed $e t a l^{16}$ found that the longer the distance travelled to primary healthcare centres in Riyadh, the lower the patients' satisfaction, with $39 \%$ of patients considering the primary healthcare centre far or very far. However, in our study, distance was not an issue, and we did not observe any association between type of healthcare facilities used or distance to healthcare facilities, and health outcomes or use of health services in KSA.

The study from Saeed et al was not the only one to focus on patients' satisfaction in KSA. Other small, nonrepresentative studies have examined patient satisfaction with healthcare facilities and services in KSA and were focused on the Saudi Ministry of Health primary healthcare centres. Most studies showed a high rate of dissatisfaction among users. Of facility characteristics, distance travelled, facility working hours, absence of specialty clinics, waiting time, waiting area structure and confidentiality measures, were the negative factors most impacting patients' satisfaction. Of staff characteristics, surgeons' services, language barriers with physicians and communication about health status, were the factors most correlated with dissatisfaction. As for patients' characteristics, women and the least educated seem to be more satisfied than men and more educated patients, respectively. ${ }^{17-19}$ Other relevant areas of healthcare services in KSA have been studied, and they also pertain to healthcare utilisation and patient satisfaction. For instance, Alahmadi ${ }^{20}$ researched patient safety culture in Saudi healthcare facilities. Interestingly, the author found that interest of management and patient safety procedures are only triggered after an adverse event has occurred. Moreover, the author notes the under-reporting of errors, even when 
Table 1 Distribution and logistic regression for diagnosis, treatment and control of hypertension by different factors; Saudis with hypertension aged 15 years or older, Kingdom of Saudi Arabia, 2013

\begin{tabular}{|c|c|c|c|c|c|c|}
\hline \multirow{2}{*}{$\begin{array}{l}\text { Sociodemographic characteristics, } \\
\text { healthcare access and diagnoses of } \\
\text { chronic conditions }\end{array}$} & \multicolumn{2}{|c|}{ Diagnosis among those hypertensive } & \multicolumn{2}{|c|}{ Treatment among those diagnosed } & \multicolumn{2}{|c|}{ Control among those treated } \\
\hline & N (weighted \%; SE) & AOR $(95 \% \mathrm{CI})$ & N (weighted \%; SE) & AOR $(95 \% \mathrm{CI})$ & N (weighted \%; SE) & AOR $(95 \% \mathrm{Cl})$ \\
\hline \multicolumn{7}{|l|}{ Sex } \\
\hline Males & $492(54.49 ; 2.21)$ & REF & $417(53.34 ; 2.37)$ & REF & 165 (49.66; 3.62) & REF \\
\hline Females & $459(45.51 ; 2.21)$ & $1.87(1.23$ to 2.85$)$ & $416(46.66 ; 2.37)$ & 1.82 (0.81 to 4.09$)$ & 195 (50.34; 3.62) & 1.61 (0.85 to 3.08$)$ \\
\hline Age (years)* & & $1.05(1.03$ to 1.07$)$ & & 1.08 (1.05 to 1.12$)$ & & 1.01 (0.99 to 1.03$)$ \\
\hline $15-24$ & $4(0.65 ; 0.33)$ & & $3(0.59 ; 0.34)$ & & $1(0.50 ; 0.50)$ & \\
\hline $25-34$ & $41(5.06 ; 0.95)$ & & $23(3.13 ; 0.84)$ & & $13(4.53 ; 1.70)$ & \\
\hline $35-44$ & $105(12.10 ; 1.42)$ & & $78(10.08 ; 1.35)$ & & $40(11.07 ; 2.08)$ & \\
\hline $45-54$ & $215(27.42 ; 2.06)$ & & $191(27.76 ; 2.21)$ & & $79(28.86 ; 3.46)$ & \\
\hline $55-64$ & $243(27.92 ; 2.06)$ & & $227(29.83 ; 2.25)$ & & 106 (30.83; 3.33) & \\
\hline $65+$ & $343(26.86 ; 1.82)$ & & $311(28.61 ; 2.01)$ & & $121(24.21 ; 2.90)$ & \\
\hline \multicolumn{7}{|l|}{ Marital status } \\
\hline Currently married & $696(78.40 ; 1.77)$ & REF & $607(77.83 ; 1.93)$ & REF & $257(74.25 ; 3.17)$ & REF \\
\hline Never married & $33(5.15 ; 1.16)$ & 0.95 (0.38 to 2.39$)$ & $22(4.41 ; 1.20)$ & 2.37 (0.58 to 9.75$)$ & $11(5.69 ; 2.05)$ & 1.73 (0.40 to 7.53$)$ \\
\hline Separated, divorced or widowed & $220(16.45 ; 1.47)$ & 0.67 (0.39 to 1.17$)$ & $202(17.76 ; 1.64)$ & $0.66(0.24$ to 1.80$)$ & $91(20.06 ; 2.74)$ & 1.88 (0.88 to 4.05$)$ \\
\hline \multicolumn{7}{|l|}{ Education } \\
\hline Primary school or less & $593(57.79 ; 2.18)$ & REF & $533(59.20 ; 2.32)$ & REF & 217 (52.12; 3.62) & REF \\
\hline Elementary or high school completed & $225(27.48 ; 2.03)$ & $1.00(0.63$ to 1.58$)$ & $195(27.10 ; 2.16)$ & 2.86 (1.16 to 7.08$)$ & $86(29.26 ; 3.48)$ & 2.25 (1.12 to 4.50$)$ \\
\hline College degree or higher education & $131(14.73 ; 1.46)$ & 1.81 (1.06 to 3.09$)$ & $104(13.70 ; 1.53)$ & 2.78 (1.14 to 6.79$)$ & $57(18.62 ; 2.77)$ & 4.63 (2.14 to 10.02$)$ \\
\hline \multicolumn{7}{|l|}{ Type of clinic last visited } \\
\hline Ministry of Health & $618(72.44 ; 2.23)$ & & $548(71.97 ; 2.40)$ & & 229 (70.65; 3.77) & \\
\hline Other governmental clinic & $70(8.89 ; 1.29)$ & & $61(8.66 ; 1.37)$ & & $31(9.69 ; 2.11)$ & \\
\hline Private clinic & $112(18.67 ; 2.03)$ & & $101(19.37 ; 2.20)$ & & $41(19.67 ; 3.52)$ & \\
\hline Distance travelled to clinic $(\mathrm{km}) \dagger$ & & 1.01 (0.99 to 1.03$)$ & & & & \\
\hline $0-2$ & $218(36.87 ; 2.66)$ & & $189(36.90 ; 2.85)$ & & $79(39.24 ; 4.50)$ & \\
\hline $3-5$ & $159(25.65 ; 2.46)$ & & $138(24.43 ; 2.56)$ & & $56(24.82 ; 4.04)$ & \\
\hline $6-10$ & $116(19.79 ; 2.22)$ & & $107(20.04 ; 2.33)$ & & $42(18.68 ; 3.40)$ & \\
\hline $11-35$ & $84(14.49 ; 1.97)$ & & $78(15.45 ; 2.21)$ & & $27(12.14 ; 2.82)$ & \\
\hline $36-100$ & $21(3.20 ; 0.86)$ & & $19(3.18 ; 0.91)$ & & $10(5.12 ; 1.90)$ & \\
\hline \multicolumn{7}{|l|}{ Diagnosis history of diabetes } \\
\hline No & $484(51.91 ; 2.23)$ & REF & $406(48.91 ; 2.39)$ & REF & $168(47.77 ; 3.62)$ & \\
\hline Yes & $462(48.09 ; 2.23)$ & 2.38 (1.60 to 3.53$)$ & $423(51.09 ; 2.39)$ & 2.21 (1.05 to 4.62$)$ & 188 (52.23; 3.62) & \\
\hline \multicolumn{7}{|l|}{$\begin{array}{l}\text { Diagnosis history of } \\
\text { hypercholesterolaemia }\end{array}$} \\
\hline No & $557(63.01 ; 2.22)$ & REF & $473(61.06 ; 2.39)$ & REF & $192(56.85 ; 3.70)$ & REF \\
\hline Yes & $322(36.99 ; 2.22)$ & 2.76 (1.72 to 4.44$)$ & $300(38.94 ; 2.39)$ & 1.78 (0.82 to 3.84$)$ & $143(43.15 ; 3.70)$ & 1.89 (1.12 to 3.18$)$ \\
\hline
\end{tabular}


Table 2 Distribution and logistic regression for diagnosis, treatment and control of diabetes by different factors; Saudis with diabetes aged 15 years or older, Kingdom of Saudi Arabia, 2013

\begin{tabular}{|c|c|c|c|c|c|c|}
\hline \multirow{2}{*}{$\begin{array}{l}\text { Sociodemographic characteristics, } \\
\text { healthcare access and diagnoses of } \\
\text { chronic conditions }\end{array}$} & \multicolumn{2}{|c|}{$\begin{array}{l}\text { Diagnosis among those } \\
\text { with diabetes }\end{array}$} & \multicolumn{2}{|c|}{ Treatment among those diagnosed } & \multicolumn{2}{|c|}{ Control among those treated } \\
\hline & N (weighted \%; SE) & AOR (95\% Cl) & $\bar{N}$ (weighted \%; SE) & AOR $(95 \% \mathrm{Cl})$ & N (weighted \%; SE) & AOR (95\% Cl) \\
\hline \multicolumn{7}{|l|}{ Sex } \\
\hline Males & 392 (61.88; 2.52) & & $378(61.40 ; 2.57)$ & & 167 (57.88; 3.92) & REF \\
\hline Females & 309 (38.12; 2.52) & & $302(38.60 ; 2.57)$ & & 149 (42.12; 3.92) & $1.50(0.76-2.97)$ \\
\hline Age (years) ${ }^{\star}$ & & 1.05 (1.03 to 1.08$)$ & & 1.03 (1.00 to 1.07$)$ & & $1.02(1.00$ to 1.05$)$ \\
\hline $15-24$ & $11(3.01 ; 1.01)$ & & $10(2.91 ; 1.01)$ & & $7(2.14 ; 1.01)$ & \\
\hline $25-34$ & $28(5.59 ; 1.29)$ & & $27(5.50 ; 1.30)$ & & $32(14.31 ; 2.95)$ & \\
\hline $35-44$ & $76(12.43 ; 1.73)$ & & $73(12.39 ; 1.77)$ & & $82(29.46 ; 3.79)$ & \\
\hline $45-54$ & 175 (29.90; 2.62) & & $168(29.69 ; 2.66)$ & & $76(28.83 ; 3.80)$ & \\
\hline $55-64$ & $182(27.80 ; 2.37)$ & & $179(28.28 ; 2.43)$ & & $114(22.71 ; 3.06)$ & \\
\hline $65+$ & $229(21.28 ; 1.95)$ & & $223(21.23 ; 1.98)$ & & $316(100.00 ; 0.00)$ & \\
\hline \multicolumn{7}{|l|}{ Marital status } \\
\hline Currently married & $535(82.08 ; 1.88)$ & & $521(82.31 ; 1.90)$ & & $65(12.60 ; 2.08)$ & REF \\
\hline Never married & $27(5.80 ; 1.41)$ & & $26(5.76 ; 1.43)$ & & $316(100.00 ; 0.00)$ & 0.35 (0.06 to 2.13$)$ \\
\hline Separated, divorced or widowed & $138(12.12 ; 1.38)$ & & $132(11.94 ; 1.40)$ & & $521(0.00 ; 0.00)$ & 0.67 (0.27 to 1.66$)$ \\
\hline \multicolumn{7}{|l|}{ Education } \\
\hline Primary school or less & $461(58.87 ; 2.62)$ & & $445(58.46 ; 2.66)$ & REF & $32(13.07 ; 2.71)$ & \\
\hline Elementary or high school completed & $172(29.59 ; 2.45)$ & & $167(29.75 ; 2.48)$ & $1.01(0.34$ to 3.00$)$ & $316(100.00 ; 0.00)$ & \\
\hline College degree or higher education & $68(11.53 ; 1.65)$ & & $68(11.79 ; 1.69)$ & 5.79 (1.57 to 21.32$)$ & $445(0.00 ; 0.00)$ & \\
\hline \multicolumn{7}{|l|}{ Type of clinic last visited } \\
\hline Ministry of Health & $469(79.94 ; 2.25)$ & REF & $454(79.71 ; 2.30)$ & REF & $225(86.23 ; 2.89)$ & REF \\
\hline Other governmental clinic & $48(8.35 ; 1.57)$ & $0.44(0.14$ to 1.34$)$ & $46(8.47 ; 1.61)$ & $4.35(0.41$ to 46.45$)$ & $16(3.96 ; 1.13)$ & $0.28(0.10$ to 0.79$)$ \\
\hline Private clinic & $59(11.70 ; 1.77)$ & 0.65 (0.28 to 1.52$)$ & $58(11.82 ; 1.81)$ & $1.00(0.17$ to 5.75$)$ & $21(9.81 ; 2.72)$ & 0.54 (0.21 to 1.43$)$ \\
\hline Distance travelled to clinic $(\mathrm{km}) \dagger$ & & 0.98 (0.96 to 1.00$)$ & & 0.97 (0.93 to 1.02$)$ & & 1.01 (0.98 to 1.04$)$ \\
\hline $0-2$ & $167(46.04 ; 3.54)$ & & $162(46.24 ; 3.59)$ & & $72(46.16 ; 5.36)$ & \\
\hline $3-5$ & $113(19.46 ; 2.51)$ & & $110(18.92 ; 2.51)$ & & $57(17.54 ; 3.09)$ & \\
\hline $6-10$ & $83(20.11 ; 2.67)$ & & $82(20.23 ; 2.71)$ & & $36(19.37 ; 4.23)$ & \\
\hline $11-35$ & 52 (11.39; 2.33) & & $51(11.55 ; 2.37)$ & & $29(16.19 ; 4.24)$ & \\
\hline $36-100$ & $6(3.00 ; 1.88)$ & & $6(3.05 ; 1.91)$ & & $2(0.74 ; 0.56)$ & \\
\hline \multicolumn{7}{|l|}{ Diagnosis history of hypertension } \\
\hline No & $432(63.73 ; 2.56)$ & REF & $419(63.96 ; 2.60)$ & & $193(61.26 ; 3.95)$ & \\
\hline Yes & 267 (36.27; 2.56) & 2.39 (1.09 to 5.25$)$ & $259(36.04 ; 2.60)$ & & $122(38.74 ; 3.95)$ & \\
\hline \multicolumn{7}{|c|}{ Diagnosis history of hypercholesterolaemia } \\
\hline No & $449(72.36 ; 2.39)$ & REF & $437(73.16 ; 2.42)$ & REF & $203(72.15 ; 3.56)$ & \\
\hline Yes & $205(27.64 ; 2.39)$ & $5.64(2.37$ to 13.44$)$ & $196(26.84 ; 2.42)$ & $0.54(0.20$ to 1.52$)$ & $97(27.85 ; 3.56)$ & \\
\hline
\end{tabular}


actual harm occurs, due to a widespread culture of blaming individual workers for errors. ${ }^{20}$

Our study has some limitations. First, our data are from a cross-sectional study, and therefore we cannot assess causality. However, our study is based on a large sample and used a standardised methodology for all its measures. Second, only $52 \%$ of respondents completed the visit to a health clinic and had their blood drawn for analysis. However, our weighting methodology accounted for this bias by applying a post-stratification adjustment using sociodemographic characteristics, health behaviours, previously diagnosed non-communicable diseases and anthropometric measurements of respondents from the household survey. Third, our study is a household survey and reflects only on individual factors that can affect access to, and utilisation of, healthcare. Also, we did not collect data on satisfaction with healthcare services. Furthermore, our study cannot assess system-based factors that affect the healthcare-seeking behaviour of the Saudi population. Such factors can be better understood through healthcare facility surveys and exit interviews with patients leaving healthcare facilities.

Despite the density of health facilities and the free healthcare system in KSA, ${ }^{1}{ }^{2}$ Saudis do not seem to seek prevention or care until after developing disease symptoms or reaching an advanced stage of illness. However, the Saudi Ministry of Health invests considerable funds in prevention and health promotion campaigns. ${ }^{21}$ It is crucial for the Ministry to understand why Saudis abstain from using preventive services, including periodic health examinations and screenings for preventable diseases. Given the lack of information on this in KSA, formative research through qualitative research methods is needed, as no assumptions exist regarding the reasons behind seeking healthcare, or the lack of care-seeking, among Saudis. However, the focus on individual factors is not enough. System-based factors should also be questioned to understand relevant aspects of the system, including the quality of care. Findings from such studies can help modify and improve the system in order to incentivise the Saudi population to benefit more from their free healthcare system.

Our findings showed a higher likelihood for women to be diagnosed with chronic conditions, such as hypertension. However, no difference exists among those diagnosed when it comes to treatment or control of the condition. Indeed, getting diagnosed requires that one actively seeks healthcare. In parallel, the health system should play an equivalent role in seeking out undiagnosed patients through organised screening programmes and protocols, such as national mass screening campaigns targeting specific diseases. Also, getting treated and having one's condition controlled once diagnosed relies more on the healthcare provider's interaction with the patient and their follow-up. Women are doing a better job seeking care, but both sexes have a long way to go to improve health and reduce burden. ${ }^{5}$ Diagnosis is the first step towards controlling a condition. Patients have to be monitored and followed to ensure proper dosage of medication and to reinforce behavioural changes. Hence, regular check-ups have to be scheduled and the patient must be reminded to come to the clinic.

Our results show the importance of health education and programmes to reach the population at home and in the workplace. Clearly, Saudis are not taking advantage of the free medical services and medications. The Saudi Ministry of Health could easily implement programmes to generate reminders for visits to clinics, which could help control diseases and reduce burden. Such systems are available in many countries, where patients are notified about their next scheduled visits. ${ }^{22}$ In KSA, such systems are more likely to be successful, as patients get their medications refilled for free from health clinics.

In parallel to understanding individual behaviour, the Saudi Ministry of Health needs to assess specific characteristics in their healthcare facilities. For instance, a targeted survey can measure the bottlenecks, including non-availability of stocks, equipment and staff, that healthcare facilities and their users face. A parallel geographically linked survey, coupled with patient exit interview surveys, could determine the impact of access and bottlenecks on the health of Saudis.

Arab culture promotes health and encourages prevention over treatment; an old Arab proverb savs, 'Prevention is better than treatment'. However, our findings do not point in this direction. Saudis seem to mostly seek healthcare when sick. Free and available healthcare services in close proximity are not enough to get Saudis to utilise the care offered. The Saudi Ministry of Health needs to do more than building additional healthcare facilities. It needs to implement a comprehensive plan that includes health education and plans to understand the barriers and bottlenecks to healthcare-seeking behaviour and access.

Acknowledgements The authors would like to thank Kate Muller at the Institute for Health Metrics and Evaluation for editing the manuscript.

Contributors AHM and CEB conceived and designed the study. MB, ZAM, MAS and MAA performed the study. AHM and CEB analysed the data. CEB, AHM, FD, MT, HK, MB, ZAM, MAS, MAA and AAAR wrote the manuscript. AAAR supervised the study. All the authors have read and approved the final manuscript.

Funding This study was financially supported by a grant from the Ministry of Health of the Kingdom of Saudi Arabia.

Competing interests None declared.

Ethics approval The Saudi Ministry of Health and its IRB have approved the study protocol. The University of Washington IRB has deemed the study as IRB exempt since the Institute for Health Metrics and Evaluation received de-identified data for this analyses.

Provenance and peer review Not commissioned; externally peer reviewed.

Data sharing statement The data collected for this study are not publicly available. Any request to access the data needs to be addressed to the Saud Ministry of Health.

Open Access This is an Open Access article distributed in accordance with the Creative Commons Attribution Non Commercial (CC BY-NC 4.0) license, which permits others to distribute, remix, adapt, build upon this work non-commercially, and license their derivative works on different terms, provided the original work is properly cited and the use is non-commercial. See: http://creativecommons.org/licenses/by-nc/4.0/ 


\section{REFERENCES}

1. Almalki M, Fitzgerald G, Clark M. Health care system in Saudi Arabia: an overview. East Mediterr Health J 2011;17:784-93.

2. Saudi Arabian Monetary Agency. Forty ninth annual report: latest economic developments. http://www.sama.gov.sa/sites/samaen/ ReportsStatistics/ReportsStatisticsLib/5600 R Annual En 49 Apx.pdf

3. El Bcheraoui C, Tuffaha M, Daoud F, et al. Low uptake of periodic health examinations in the Kingdom of Saudi Arabia, 2013. J Fam Med Prim Care Accepted March 2015. In press.

4. El Bcheraoui C, Basulaiman M, Wilson S, et al. Breast cancer screening in Saudi Arabia: free but almost no takers. PLOS ONE 2015;10:e0119051.

5. El Bcheraoui C, Memish ZA, Tuffaha M, et al. Hypertension and its associated risk factors in the Kingdom of Saudi Arabia, 2013: a national survey. Int J Hypertens 2014;2014:564679.

6. El Bcheraoui C, Basulaiman M, Tuffaha M, et al. Status of the diabetes epidemic in the Kingdom of Saudi Arabia, 2013. Int $J$ Public Health 2014;59:1011-21.

7. Buzza C, Ono SS, Turvey $\mathrm{C}$, et al. Distance is relative: unpacking a principal barrier in rural healthcare. J Gen Intern Med 2011;26(Supp 2):648-54.

8. Ward B, Humphreys J, McGrail M, et al. Which dimensions of access are most important when rural residents decide to visit a general practitioner for non-emergency care? Aust Health Rev 2015;39:121-6.

9. Yamauchi Y, Fujiwara T, Okuyama M. Factors influencing time lag between initial parental concern and first visit to child psychiatric services among ADHD children in Japan. Community Ment Health $J$ 2014. Published Online First: 23 December 2014. doi:10.1007/ s10597-014-9803-y

10. Schoeps A, Gabrysch S, Niamba L, et al. The effect of distance to health-care facilities on childhood mortality in rural Burkina Faso. Am J Epidemiol 2011;173:492-8.

11. Centers for Disease Control and Prevention. National Health and Nutrition Examination Survey: Health Tech/Blood Pressure Procedures Manual. cdc.gov. 2009. http://www.cdc.gov/nchs/data/ nhanes/nhanes_09_10/BP.pdf (accessed 11 Dec 2014).
12. U.S.-Saudi Arabian Business Council. Saudi Arabia's 2014 budget emphasizes long-term development. U.S.-Saudi Arabian Business Council. 2013. http://www.us-sabc.org/custom/news/details.cfm? id=1541\#.VLROoivF98M

13. Vanderschuren M, McKune D. Emergency care facility access in rural areas within the golden hour?: Western Cape case study. Int $J$ Health Geogr 2015;14:5

14. Gonçalves J, Ferreira JA, Condessa B. Making regional facility location decisions: the example of Hospital do Oeste Norte, Portugal. Geospat Health 2014;9:1-6.

15. Murad AA. Using geographical information systems for defining the accessibility to health care facilities in Jeddah City, Saudi Arabia. Geospat Health 2014;8:295.

16. Saeed AA, Mohammed BA, Magzoub ME, et al. Satisfaction and correlates of patients; satisfaction with physicians; services in primary health care centers. Saudi Med J 2001;22:262-7.

17. Al-Doghaither AH. Inpatient satisfaction with physician services at King Khalid University Hospital, Riyadh, Saudi Arabia. East Mediterr Health J 2004;10:358-64.

18. El Shabrawy MA, Mahmoud ME. A study of patient satisfaction with primary health care services in Saudi Arabia. J Community Health 1993;18:49-54.

19. Qatari GA, Haran D. Determinants of users' satisfaction with primary health care settings and services in Saudi Arabia. Int $J$ Qual Health Care 1999;11:523-31.

20. Alahmadi HA. Assessment of patient safety culture in Saudi Arabian hospitals. Qual Saf Health Care 2010;19:1-5.

21. Saudi Ministry of Health. Awareness Campaigns. Kingdom of Saudi Arabia-Ministry of Health Portal. http://www.moh.gov.sa/en/ HealthAwareness/Campaigns/Pages/default.aspx (accessed 12 Jan 2015)

22. Agrawal A, Mayo-Smith MF. Adherence to computerized clinical reminders in a large healthcare delivery network. In: Fieschi M, ed. Medinfo. Amsterdam: IOS Press, 2004:111-14 